\title{
High incidence of monozygotic twinning in infertility treatment
}

\author{
Ales Sobek Jr. ${ }^{\mathrm{a}, \mathrm{c}}$, Martin Prochazka ${ }^{\mathrm{b}}$, Eva Klaskovac, ${ }^{\mathrm{c}}$, Marek Lubusky ${ }^{\mathrm{a}}$, Radovan Pilka ${ }^{\mathrm{a}}$
}

Background. Monozygotic twinning is associated with increased perinatal morbidity and mortality. There is evidence that the number of monozygotic twins increases after assisted reproductive techniques.

Methods. We searched PUBMED, MEDLINE, and Scopus from 1987 to 2015 for studies analyzing the incidence and possible etiology of monozygotic twinning in infertility patients and critically reviewed the current state of knowledge. Results and Conclusions. Monozygotic twinning is a rare in natural conception but occurs around twice the normal rate after assisted reproduction. Factors associated with this phenomenon remain speculative, though there is some evidence that micromanipulation techniques, prolonged culture, and genetics are involved. In view of the possible complications, adequate pre-conception counselling is advocated.

Key words: monozygotic twins, infertility, incidence, risk factors

Received: December 1, 2015; Accepted with revision: March 15, 2016; Available online: April 1, 2016 http://dx.doi.org/10.5507/bp.2016.016

${ }^{a}$ Department of Obstetrics and Gynecology, University Hospital Olomouc and Faculty of Medicine and Dentistry, Palacky University Olomouc, Czech Republic

${ }^{b}$ Department of Medical Genetics, University Hospital Olomouc and Faculty of Medicine and Dentistry, Palacky University Olomouc, Czech Republic

'Fertimed Infertility Center, Boleslavova 2, 77200 Olomouc, Czech Republic

${ }^{d}$ Department of Paediatrics, University Hospital Olomouc and Faculty of Medicine and Dentistry, Palacky University Olomouc, Czech Republic

Corresponding author: Martin Prochazka, e-mail: martin.prochazka@fnol.cz

\section{INTRODUCTION}

It is well known that multiple births occur more often after assisted reproductive technologies (ART) than after spontaneous conceptions. The higher incidence of dizygotic twins arising from the transfer of two embryos is an understandable consequence of ART but the mechanism of monozygotic twinning after ART is still unclear. Monozygotic twins occur when the fertilization of one oocyte by one sperm produces genetically identical twins. Monozygotic twinning (MZT) is a relatively rare phenomenon, with an incidence of about $1 \%$ of natural conceptions. In assisted conceptions, the risk of monozygotic twinning has been estimated to be about twice as high ${ }^{1}$. Multiple births are generally associated with many maternal and fetal complications, which are more severe in MZT that share a single placenta. Therefore, understanding the mechanism of increased monozygotic twinning in ART is important and could minimize the incidence of such high-risk pregnancies. We reviewed the scientific literature on the etiology, frequency, risk factors, and complications associated with MZT in patients treated with ART.

\section{MECHANISM OF MONOZYGOTIC TWINNING}

Monozygotic twins arise from one zygote which divides into two separate individuals. The time at which the embryo divides is a critical factor in subsequent placental development, and the risk of complications is related to placental sharing ${ }^{2}$. Division on days 1-4 (morula), before

\begin{tabular}{|l|c|c|c|}
\hline $\begin{array}{l}\text { Days after } \\
\text { fertilization }\end{array}$ & $1-4$ & $4-8$ & $8-12$ \\
\hline $\begin{array}{l}\text { Twins } \\
\text { produced }\end{array}$ & dichorionic-diamniotic & monochorionic-diamniotic & monochorionic-monoamniotic \\
\hline
\end{tabular}

Fig. 1. Type of monozygotic twins depends on the time of division. 
the chorion has differentiated, results in dichoriotic-diamniotic twins $(20 \%)$, which are indistinguishable from dizygotic twins resulting from two embryos. Division on 4-8 days (blastocyst) results in monochoriotic-diamniotic twins (75\%), and division on 1-2 weeks results in monochoriotic-monoamniotic twins, which share one placenta and one amniotic sac. Conjoined twins result from division after day 12 .

Monozygotic twinning has been induced experimentally in animal models. In 1921, Stockard ${ }^{3}$ demonstrated an increased incidence of MZT in fish after either the available oxygen or the incubation temperature was reduced. Similarly, increased MZT has been identified in mice under in vitro conditions, although mice do not normally show MZ twinning in natural cycles ${ }^{4}$. MZT were also induced by delayed fertilization in rabbits ${ }^{5}$. In vitro conditions also seem to be related to increased monozygotic twinning in humans. The reason for this phenomenon has been discussed for almost three decades and several potential mechanisms have been proposed. Earlier studies examined the effect of ovulation induction, whereas later research concentrated on the effects of micromanipulation techniques and the length of culture. Recent studies have identified a possible genetic etiology ${ }^{6}$. The rate of monozygotic twinning after natural conception varies only slightly in the literature, from $0.4 \%$ (ref. $^{1}$ ) to $1.6 \%$ (ref. ${ }^{7}$ ). In contrast, studies of assisted conceptions show wider variance, ranging from $0.72 \%$ (ref. $^{8}$ ) to $12.5 \%$ (ref. $^{9}$ ). Generally, the incidence of monozygotic twinning after assisted reproductive techniques is about twice as high as after natural conceptions, although some large studies have detected no association between MZ twinning and infertility treatment ${ }^{10}$.

\section{Micromanipulation techniques}

The routine application of intracytoplasmic sperm injection (ICSI) and assisted hatching (AH) has prompted discussion of a possible connection between micromanipulation techniques and increased MZT. To successfully implant into the uterine wall, an embryo must hatch out of the zona pellucida (ZP), a protein layer covering the embryo in the initial stages of development, and attach to the inner lining of the uterus. Both techniques (ICSI and $\mathrm{AH})$ manipulate the ZP in different ways. ICSI involves direct injection of a spermatozoon into the oocyte. The technique requires a very small number of sperm and allows the use of sperm with limited motility. The other technique, AH, involves mechanical, chemical, or laser incision of the ZP of a fertilized embryo. AH has developed based on the observation that embryos with a thin ZP have a higher rate of implantation after in vitro fertilization (IVF). Both of these techniques (ICSI and AH) leave small defects in the ZP, which may complicate the natural process of embryo hatching. The embryo may bypass its own mechanism of ZP lysis and herniate through these defects, thus resulting in MZT (ref. ${ }^{11}$ ). If so, the size of the defect will probably affect the rate of MZT. There is a distinct difference in the size of the artificial breach in the ZP when ICSI and AH are used. The zona opening formed by $\mathrm{AH}$ is $25-30 \mu \mathrm{m}$ in diameter, whereas the puncture site following ICSI is much smaller $(7-8 \mu \mathrm{m}$ in diameter) (ref. ${ }^{12}$ ). In theory, a smaller hole would not allow the embryo to hatch appropriately and MZT would be more common in ICSI than AH patients. This finding is in agreement with the results of Vitthala ${ }^{11}$, who collected data from 27 studies in a meta-analysis, and concluded that couples who underwent ICSI had a higher MZT rate than couples who underwent conventional IVF or AH. In contrast, Luke et al. ${ }^{2}$ reported a greater effect after AH, although only in 2-3 day embryos (cleavage stage). The same observation was recently reported by Kanter et al. ${ }^{13}$, who showed that early stage embryos may be more vulnerable to the effect of AH than blastocysts. Unlike the previous results, there are also reports, although involving a smaller number of patients, which show that micromanipulation techniques have no effect on MZT (ref. ${ }^{14,15}$ ). It is apparent that larger studies with statistical power are needed to determine whether or not micromanipulation of the ZP increases the rate of monozygotic pregnancies in IVF patients.

\section{Length of culture}

It has previously been reported that culture to the blastocyst stage (day 5 ) in women with good-quality embryos may facilitate embryo selection, reduce aneuploidy embryos $^{16}$ and improve live birth rates ${ }^{17}$. Rijnders ${ }^{18}$ and Peramo ${ }^{19}$ were the first to report an association between the incidence of MZT and blastocyst transfer. They observed a significant difference between the MZT rate after embryo transfer (ET) on day $3(0.68 \%)$ and transfer on day 5 (2.7\%). Da Costa ${ }^{20}$ reported that $3.9 \%$ of pregnancies generated by blastocyst transfer were complicated by MZT, and Behr $^{21}$ detected an incidence of $5 \%$. Wrigth et al. ${ }^{22}$ examined the 1999-2000 data from the American Society of Assisted Reproductive Technologies (SART) for 39,198 pregnancies. The incidence of MZT was four-fold higher after embryo transfer on day 5 than after embryo transfer on day 3. Similarly, a more recent and larger study that examined data from the SART showed that MZT was more likely to develop from embryos transferred on days 5-6 than from cleavage embryos ${ }^{2}$.

Conversely, Franasiak et al. ${ }^{23}$ recently reported that transfer in the blastocyst stage is not associated with increased MZT rates when controlling for embryo quality based on a cohort of 342 monozygotic pregnancies.

To explain the possible mechanism by which prolonged cultivation affects the MZT rate, most theories center on hardening of the ZP ( ref. $^{20}$ ). Zona hardening may squeeze the inner cell mass (ICM) and induce embryo splitting during hatching ${ }^{24}$. Increased MZT might also correlate with the transfer of high-quality embryos, which are more often transferred after prolonged culture. These embryos are more sensitive to the effects of mechanical manipulation in the laboratory or to changes in temperature and $\mathrm{pH}$ during monitoring ${ }^{2}$, which might result in higher rates of MZT after blastocyst transfer.

\section{Culture medium conditions}

Studies of animals have shown that mice blastocysts duplicate their ICM more frequently in vitro than in 
vivo $^{25}$. A similar phenomenon can be expected in humans. Steinman ${ }^{26}$ speculated that the prolonged exposure of blastocysts to lower calcium levels in the culture medium could enhance ICM division because the intercellular bonds are destabilized. Others believe that changes in the culture medium, including an absence of growth factors and a higher glucose content, could produce free radicals, which induce apoptosis, leading to the disruption of the ICM and presumably zygotic splitting ${ }^{22}$. Similarly, Behr ${ }^{21}$ suggested that the current culture media cause perturbations of the cell-to-cell adhesions, facilitating the splitting of the ICM. Several researchers also detected a possible association between temperature changes and a higher incidence of MZT in animal models ${ }^{3}$. However, no association has been demonstrated between the transfer of thawed embryos and monozygotic twinning in humans ${ }^{27}$.

\section{Genetics}

Autosomal dominant inheritance with reduced penetrance has been proposed as the possible etiology for familial MZT. Inheritance has been reported to occur through the maternal and paternal lines ${ }^{28}$. Hamamy ${ }^{29}$ reported a high incidence of MZT $(n=13)$ in an extended Jordanese family. Shapiro ${ }^{30}$ investigated 10 families with multiple pairs of monozygotic twins born to parents who were also born as monozygotic twins. In a recent study, Sobek et al. ${ }^{6}$ documented that $45 \%$ of the group of women who had monozygotic twins also had a family history of MZT. The results of those studies suggested that the incidence of MZT might be under the control of hereditary factors with genes transferred similarly by both the male and female parents. In the past, the transfer of an increased number of embryos was common in an effort to increase fertility rates. In studies reporting a higher incidence of monozygotic twins, the average rate was 2-4 embryos/ET (3.6 embryos/ET ${ }^{12}, 3.2 / \mathrm{ET}^{24}$, and 2.8/ET ${ }^{8}$ ). Theoretically, if some of the embryos were "genetically" prone to produce MZT, and more of such embryos were implanted, MZT might have been increased after IVF in those studies than the normal population.

\section{Ovulation induction}

Derom et al. ${ }^{1}$ reported an increased incidence of MZT in patients after the induction of ovulation with gonadotrophins or clomifen citrate. They believed that medication can alter the structure of the ZP, making the embryo more vulnerable to ICM splitting.

\section{Age}

Later maternal age is considered by many as the only factor that increases the frequency of monozygotic twinning in natural cycles ${ }^{31}$ A gradual reduction in the thickness of the ZP with increasing age in women has been reported $^{32}$. A thinner $\mathrm{ZP}$ could be more vulnerable to inner cell protrusion at multiple sites during zona lysis, facilitating the division of the ICM. In contrast, a recent study by Knopman et al. ${ }^{27}$ found increased MZT rates in women < 35 years old. However, it must be noted that the rate of blastocyst transfer, which is believed to increase monozygotic twinning, is higher in younger women with good-quality embryos than in women over 35 years old, which could confound these results.

\section{Preimplantation genetic diagnosis (PGD)}

Verpoest et al. ${ }^{33}$ were the first to report the incidence of MZT after preimplantation genetic diagnosis (PGD). They assumed that the incidence of MZT was increased by breaks in the ZP associated with blastomere biopsy, similar to those formed by micromanipulation techniques. They found higher a incidence in the group treated with PGD than in the group treated without it, but the difference was not significant $(2.1 \%$ vs $1.5 \%$, respectively).

\section{Complications of monozygotic twins}

Multiple pregnancies are associated with an increased risk of maternal and fetal complications. Women carrying twins more frequently suffer from nausea, hypertension, and pre-eclampsia. Multiple pregnancies incur a higher risk of perinatal morbidity, mortality, prematurity, and growth restrictions. MZT pregnancies are associated with a perinatal mortality rate at least three-fold higher than that associated with dizygotic twin pregnancies ${ }^{34}$, and the twins are also at greater risk of perinatal morbidity associated with prematurity than are dizygotic twins. They display higher rates of fetal abnormalities, including neural-tube defects, congenital heart diseases, limb reduction defects, and deformities. The risk of congenital anomalies in MZT is about $10 \%\left(\right.$ ref. $\left.^{5}\right)$. The placental arrangement in monozygotic monochorionic twins poses additional risks. Monochorionic twins share one placenta and tend to suffer hemodynamic complications. These include twin-totwin transfusion syndrome (TTTS), twin embolization syndrome $(0.1 \%)$, reversed arterial perfusion syndrome, umbilical knots, and thrombosis. TTTS is the commonest disorder and occurs in response to unbalanced vascular communication in the placenta. Vascular anastomosis are present in more than $90 \%$ of monochorionic pregnancies ${ }^{35}$, but do not usually cause problems. When the placenta is unequally shared, blood can be transfused disproportionately from one twin (the donor) to the other twin (the recipient). This transfusion causes the donor twin to lose blood volume, whereas the recipient twin must deal with an overload of blood. The excess blood exerts abnormal strain on the heart of the recipient fetus, causing the development of polyhydramnion and eventual heart failure. In the donor twin, the loss of blood leads to slower growth, and poor urinary output, causing oligo- or anhydramnion. TTTS is a severe complication in MZT, accounting for $10 \%-16 \%$ of perinatal mortality ${ }^{36}$.

\section{DISCUSSION}

The major limitation in the research into MZT is the extremely low incidence of monozygotic twinning, even in patients treated for infertility. Monozygotic twins are a rare phenomenon and very large studies would be required to obtain satisfactory statistical power. Studies of the effects of culture conditions are limited because the media used, the sources of mineral oil, and the plastic- 
ware used change over time. They can also be obtained from different suppliers, which makes any comparison even more difficult. Another factor limiting the accuracy of the studies is the actual identification of monozygotic twins. The only reliable identification of monozygotic twins is by ultrasound after single-embryo transfer or by DNA analysis of the twins born when more than one embryo was transferred. Most studies identify MZT from first-trimester ultrasound data based on the number of fetuses present relative to the number of embryos transferred, which identifies approximately two thirds of MZT (ref. ${ }^{11}$ ). The reasons for the higher incidence of MZT after ART most commonly discussed in the literature are micromanipulation techniques, blastocyst transfer, genetics, maternal age, and culture medium and conditions. Most of the larger studies and meta-analyses agree on a possible association between prolonged culture or micromanipulation techniques and the higher incidence of monozygotic twinning in women treated for infertility. There is also growing evidence of genetic background of this phenomenon.

\section{CONCLUSION}

MZT is a rare phenomenon that occurs more often after assisted conception than natural conception. The reasons for the increased MZT in IVF patients have been widely discussed but no general consensus has been achieved. The major limitation of studies on MZT is the very low incidence of MZT in natural and IVF cycles. Thus, only large sample size studies provide sufficient statistical power. The current data show that several factors might contribute to the overall increased rate of MZT using assisted reproductive techniques. The majority of larger studies agree on the possible impact of micromanipulation techniques and prolonged culture, some studies suggest genetic influences but the results differ. In view of the complications arising from MZT pregnancies, proper counselling of patients before infertility treatment and examination of chorionicity in the early weeks of gestation are essential.

\section{ABBREVIATIONS}

AH: Assisted hatching; ART: Artificial reproductive technology; ET: Embryo-transfer; ICSI: Intracytoplasmic sperm injection; ICM: Inner cell mass; IVF: In vitro fertilization; MZT: Monozygotic twinning; TTTS: Twin-to-twin transfusion syndrome; PGD: Preimplantation genetic diagnosis; ZP: Zona pellucida.

Author contributions: AS, EK: literature search; AS: manuscript writing; MP, ML, RP: final approval.

Conflict of interest statement: None declared.

\section{REFERENCES}

1. Derom C, Vlietinck R, Derom R, Van den Berghe $H$, Thiery $M$. Increased monozygotic twinning rate after ovulation induction. Lancet 1987;1:1236-8.

2. Luke B, Brown MB, Wantman E, Stern JE. Factors associated with monozygosity in assisted reproductive technology pregnancies and the risk of recurrence using linked cycles. Fertil Steril 2014;101(3):683-9.

3. Stockard CR. Developmental rate and structural expression: an experimental study of twins, double monsters and single deformities, and the interaction among embryonic organs during their origin and development. American Journal of Anatomy 1921;28:115-262.

4. McLaren A, Molland P, Signer E. Does monozygotic twinning occur in mice? Genetical Research 1995;66:195-202.

5. Hall JG. Twinning. Lancet 2003;362:735-43.

6. Sobek A Jr, Zbořilová B, Procházka M, Šilhánová E, Koutná O, Klásková E, Tkadlec E, Sobek A. High incidence of monozygotic twinning after assisted reproduction is related to genetic information, but not to assisted reproduction technology itself. Fert Ster 2015;3:756-60.

7. Pinborg A, Loft A, Rasmussen S, Andersen N. Hospital care utilization of IVF/ICSI twins followed until 2-7 years of age: a controlled Danish national cohort study. Hum Reprod 2004;19:2529-36.

8. Schachter M, Raziel A, Friedler S, Strassburger D, Bern O, Ron-El R. Monozygotic twinning after assisted reproductive techniques: a phenomenon independent of micromanipulation. Hum Reprod 2001;16:1264-9.

9. Tarlatzis B, Qublan H, Sanopoulou T, Zepiridis L, Brimbizis B, Bontis $J$. Increase in the monozygotic twinning rate after intracytoplasmic sperm injection and blastocyst stage embryo transfer. Fertil Steril 2002;77:196-8.

10. Zhu JL, Basso 0, Obel C, Christensen K, Olsen J. Infertility, infertility treatment and twinning: the Danish National Birth Cohort Human Reprod 2007;4:1086-90.

11. Vitthala S, Gelbaya1 TA, Brison DR, Fitzgerald CT, Nardo LG. The risk of monozygotic twins after assisted reproductive technology: a systematic review and meta-analysis. Hum Rep Update 2009;15(1):45-55.

12. Sills ES, Moomjy M, Naninovic N, Veeck L, McGee M, Palermo GD, Rosenwaks Z. Human zona pellucida micromanipulation and monozygotic twinning frequency after IVF. Hum Reprod 2000;15:890-5.

13. Kanter JR, Boulet SL, Kawwass JF, Jamieson DJ, Kissin DM. Trends and correlates of monozygotic twinning after single embryo transfer. Obstet Gynecol 2015;1:111-7.

14. Elizur SE, Levron J, Shrim A, Sivan E, Dor J, Shulman. Monozygotic twinning is not associated with zona pellucida micromanipulation procedures but increases with high-order multiple pregnancies. Fertil Steril 2004;2:500-1.

15. Milki AA, Jun SH, Behr B, Giudice LC, Westphal LM. Incidence of monozygotic twinning with blastocyst transfer compared to cleavage-stage transfer. Fertil Steril 2003;79:504-6.

16. Adler A, Lee H-L, McCulloh DH, Ampeloquio E, Clarke-Williams M, Wertz BH. Blastocyst culture selects for euploid embryos: comparison of blastomere and trophectoderm biopsies. Reprod Biomed Online 2014;28:485-91.

17. Glujovsky D, Blake D, Farquhar C, Bardach A. Cleavage stage versus blastocyst stage embryo transfer in assisted reproductive technology. The Cochrane Database of Systematic Reviews. 2012, Issue 7. Art. No.: CD002118. doi: 10.1002/14651858.CD002118.pub4

18. Rijnders PM, van Os HC, Jansen CAM. Increased incidence of monozygotic twinning following the transfer of blastocysts in human IVF/ ICSI. Fertil Steril 1998;70(1):15-6.

19. Peramo B, Ricciarelli E, Cuadros-Fernandez JM, Huguet E, Hernandez ER. Blastocyst transfer and monozygotic twinning. Fertil Steril 1999;72:1116-7.

20. da Costa AA, Abdelmassih S, de Oliveira FG, Abdelmassih V, Abdelmassih R, Nagy ZP. Monozygotic twins and transfer at the blastocyst stage after ICSI. Hum Reprod 2001;16:333-6.

21. Behr B, Fisch JD, Racowsky C, Miller K, Poole R, Milki AA. Blastocyst-ET and monozygotic twinning. J Assist Reprod Genet 2000;17:349-51.

22. Wright V, Schieve LA, Vahratian A, Reynolds MA. Monozygotic twinning associated with day 5 embryo transfer in pregnancies conceived after IVF. Hum Reprod 2004;19:18316.

23. Franasiak JM, Dondik Y, Molinaro TA, Hong KH, Forman EJ, Werner MD, Upham KM, Scott RT Jr. Blastocyst transfer is not associated with 
increased rates of monozygotic twins when controlling for embryo cohort quality. Fertil Steril 2015;1:95-100.

24. Alikani M, Cekleniak NA, Walters E, Cohen J. Monozygotic twinning following assisted conception: an analysis of 81 consecutive cases. Hum Reprod 2003;18:1937-43.

25. Hsu YC, Gonda MA. Monozygotic twin formation in mouse embryos in vitro. Science 1980;209:605-6.

26. Steinman G. Mechanisms of twinning VI. Genetics and the etiology of monozygotic twinning in in vitro fertilization. Journal of Reproductive Medicine 2003;48:583-90.

27. Knopman J, Krey LC, Lee J, Fino E, Novetsky AP, Noyes N. Monozygotic twinning: an eight-year experience at a large IVF center. Fertil Steri 2010;94:502-10.

28. Harvey M, Huntley R, Smith D. Familial monozygotic twinning. The Journal of Pediatrics 1977;90:246-9.

29. Hamamy HA, Ajlouni HK, Ajlouni KM. Familial monozygotic twinning: report of an extended multi-generation family. Twin Research 2004; 7:219-22.
30. Shapiro LR, Zemek L, Shulman MJ. Genetic aethiology for monozygotic twinning. Birth defects. Orig Art Series 1978;14:219-25.

31. Bulmer MG. The Biology of Twinning in Man. Oxford, UK: Oxford University Press, 1970.

32. Cohen J, Feldberg D. Effects of the size and number of zona pellucida openings on hatching and trophoblast outgrowth in the mouse embryo. Mol Report Dev 1991;30:70-8.

33. Verpoest W, Landuyt LV, Desmyttere S, Cremers A, Devroey P, Liebaers I. The incidence of monozygotic twinning following PGD is not increased. Hum Rep 2009;24(11):2945-50.

34. Toledo M. Is there increased monozygotic twinning after assisted reproductive technology? Aust N Z J Obstet Gynaecol 2005;45:360-4

35. Kim JA, Jeong Yeon Cho, Kyeong Chun. Complications Arising in Twin Pregnancy: Findings of Prenatal Ultrasonography. Korean J Radiol 2003;4:54-60.

36. De Villiers SF, Zhao DP, Cohen D, van Zwet EW, Duan T, Oepkes $\mathrm{D}$, Lopriore E. Correlation between veno-venous anastomoses, TTTS and perinatal mortality in monochorionic twin pregnancies. 2015;5:603-6. 